\title{
Two-electron photo-oxidation of betanin on titanium dioxide and po- tential for improved dye-sensitized solar energy conversion
}

\author{
Fritz J. Knorr, ${ }^{a}$ Deborah J. Malamen, ${ }^{a}$ Jeanne L. McHale, ${ }^{*} a$ \\ Arianna Marchioro, ${ }^{b}$ and Jacques-E. Moser ${ }^{b}$ \\ ${ }^{a}$ Department of Chemistry, Washington State University Box 644630 \\ Pullman, WA 99164-4630 USA \\ ${ }^{b}$ Photochemical Dynamics Group, Institute of Chemical Sciences and Engineering \\ École Polytechnique Fédérale de Lausanne \\ CH-1015 Lausanne, Switzerland
}

\begin{abstract}
The plant pigment betanin is investigated as a dye-sensitizer on $\mathrm{TiO}_{2}$ with regard to its potential to undergo twoelectron oxidation following one-photon excitation. Electrochemical, spectroelectrochemical and transient absorption measurements provide evidence for two-electron proton-coupled photo-oxidation leading to a quinone methide intermediate which rearranges to 2-decarboxy-2,3-dehydrobetanin. Time-resolved spectroscopy measurements of betanin on nanocrystalline $\mathrm{TiO}_{2}$ and $\mathrm{ZrO}_{2}$ films were performed on femtosecond and nanosecond time-scales and provide evidence for transient species with absorption bands in the blue and the red. The results shed light on previous reports of high quantum efficiencies for electron injection and point the way to improved solar conversion efficiency of organic dyesensitized solar cells.
\end{abstract}

Keywords: $\mathrm{TiO}_{2}$, betanin, interfacial electron transfer, solar energy

\section{INTRODUCTION}

Dye-sensitized solar energy conversion based on earth abundant metal oxides, especially $\mathrm{TiO}_{2}$, is potentially an economical and environmentally friendly means to convert sunlight into electricity. ${ }^{1,2}$ However, reliance on synthetic organic and metallorganic dyes as sensitizers offsets these advantages, especially in the case of widely used ruthenium containing dyes. The advantageous long term stability of Ru-based sensitizers derives from the facile one-electron oxidation and regeneration of the $\mathrm{Ru}^{2+}$ center. All-organic molecules, on the other hand, are prone to proton-coupled twoelectron oxidation which may lead to undesirable bond-breaking which hinders regeneration. In this work, we investigate a plant pigment, betanin, which evidences enhanced quantum efficiency for converting photons to electrons as a result of two-electron oxidation at the aromatic hydroxyl group.

We reported the first betanin-based dye-sensitized solar cell (DSSC) in $2008,{ }^{3}$ and more recently we presented evidence for incident photon-to-current conversion efficiencies (IPCEs) which at their maxima exceed $100 \%$ after correction for reflection/absorption losses of the conductive glass. ${ }^{4}$ The betalain family of plant pigments, which includes betanin from red beet root and cactus pears, has attracted the interest of several groups, ${ }^{5,6,7}$ who in agreement with our work find fairly high IPCE, good photocurrents, but low overall energy conversion efficiency as a result of low photovoltages. For example, our best betanin-based DSSCs give photocurrents as high as $14 \mathrm{~mA} / \mathrm{cm}^{2}$, energy conversion efficiencies of $2.7 \%$, and uncorrected IPCE maxima near $100 \%$. However, the open-circuit voltage $V_{O C}$ is typically less than $0.4 \mathrm{~V}$, much less than the thermodynamic maximum of about $1 \mathrm{~V}^{8}{ }^{8}$ Since the maximum voltage is limited by recombination, and IPCE maxima near $100 \%$ would preclude recombination if one photon leads to one injected electron,

\footnotetext{
*Corresponding author, jmchale@wsu.edu
}

Physical Chemistry of Interfaces and Nanomaterials XIII, edited by Natalie Banerji,

Sophia C. Hayes, Carlos Silva, Proc. of SPIE Vol. 9165, 91650N · ( 2014 SPIE

CCC code: $0277-786 X / 14 / \$ 18 \cdot$ doi: $10.1117 / 12.2063888$ 
we hypothesize that betanin undergoes two-electron oxidation from its excited electronic state when adsorbed on nanocrystalline $\mathrm{TiO}_{2}$.

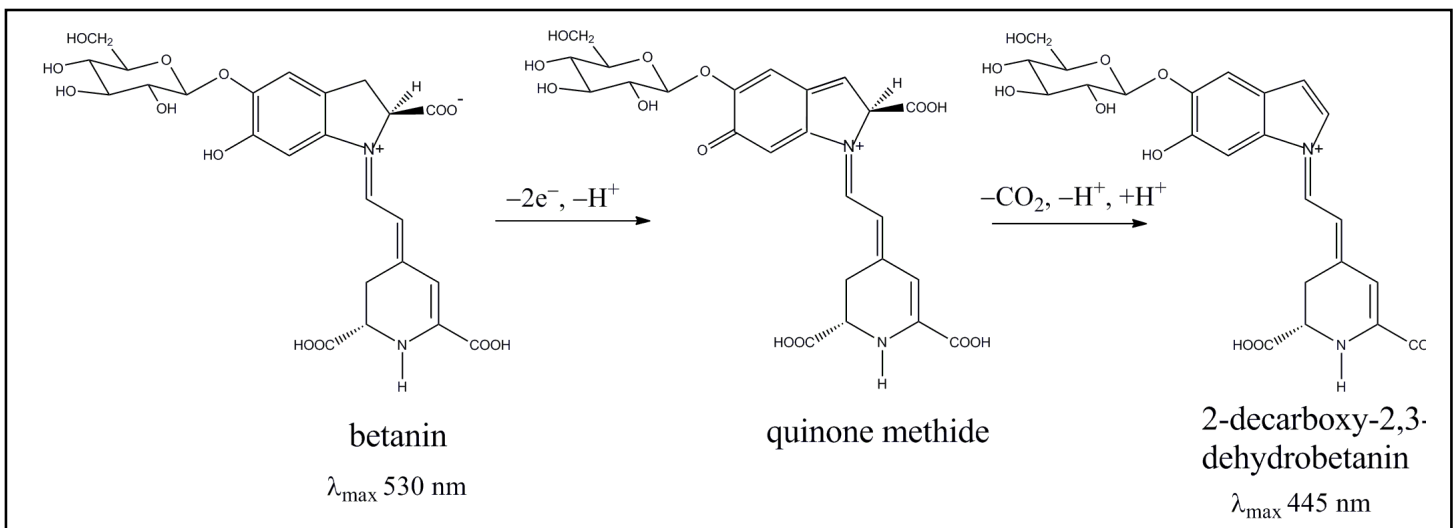

Fig. 1 Betanin and its two-electron oxidation product, a quinone methide, which rearranges to 2-decarboxy2,3-dihydrobetanin on loss of $\mathrm{CO}_{2}$. Scheme is adapted from Ref. 10

The food science community has had a long-standing interest in anti-oxidant activity of betalains, and it is known that this activity is correlated to the presence of phenolic hydroxyl groups. ${ }^{9}$ Wybraniec et al. ${ }^{10,11,12}$ have studied the oxidation of betanin $(\mathrm{Bt})$ and its aglycone betanidin $(\mathrm{Bd})$. They report that in the $\mathrm{pH}$ range 3 to 5 , electrochemical oxidation of $\mathrm{Bd}$ is a reversible two-electron, one-proton process, while at somewhat higher $\mathrm{pH}$ this changes to a twoelectron, two-proton process. Oxidization of Bt by the radical anion of ABTS, 2,2'-azinobis(3-ethylbenzthiazoline-6sulfonic acid), was reported to result in the two-electron oxidation product shown in Fig. 1, a quinone methide (Qm), which rearranges to 2-decarboxy-2,3-dehydrobetanin $(\mathrm{dhBt})$. The semiquinone radical of Bt, the one-electron oxidation product, was presumed to be an intermediate. The enzymatic oxidation of $\mathrm{Bt}$, on the other hand, was observed to take place by a single-step, two-electron process that also resulted in the formation of dhBt, a stable yellow compound with wavelength of maximum absorption $\lambda_{\max }$ at $445 \mathrm{~nm}$. Thus there is evidence that Bt is capable of single-step, two-electron oxidation in aqueous media, but the mechanism of photo-oxidation of the adsorbed molecule on $\mathrm{TiO}_{2}$ has not been investigated until now.

In this work, we pursue the electrochemistry of $\mathrm{Bt}$ in aqueous solution as a function of $\mathrm{pH}$, spectroelectrochemistry of $\mathrm{Bt}$ on a nanocrystalline $\mathrm{TiO}_{2}$ electrode in contact with nonaqueous electrolyte, and transient absorption spectra of $\mathrm{Bt}$ on $\mathrm{TiO}_{2}$ on femtosecond and nanosecond time scales. The results point the way to improved efficiency of solar energy conversion using sustainable plant-based sensitizers.

\section{EXPERIMENTAL}

Betanin was extracted from red beet root and subjected to medium-pressure liquid chromatography (MPLC) as described in Ref. 4, except that a masticating juicer rather than extraction was used to obtain the beet juice. The purple fraction from MPLC was freeze-dried and the resulting powder subjected to recrystallization from $10 \%$ methanol, $0.1 \%$ $\mathrm{HCl}$ in water at $-10{ }^{\circ} \mathrm{C}$. HPLC confirmed that the resulting fuschia fraction was composed of the pigments betanin and its $\mathrm{C}-15$ epimer isobetanin.

The voltammetric results were measured on a $0.1 \mathrm{mM}$ solution of betanin in degassed $0.2 \mathrm{M} \mathrm{NaClO}_{4}$ supporting electrolyte. The $\mathrm{pH}$ was adjusted with $\mathrm{HCl}$. Digital pulsed voltammetry (DPV) was measured with a BAS 100A electrochemical analyzer. The working electrode was a $3 \mathrm{~mm}$ glassy carbon electrode. The working electrode was polished with $1 \mu \mathrm{m}$ alumina between measurements. The reference electrode was $\mathrm{Ag} / \mathrm{AgCl}$ and the counter electrode was Pt wire. The scan rate was $4 \mathrm{mV} / \mathrm{s}$, pulse amplitude $50 \mathrm{mV}$, pulse width $50 \mathrm{~ms}$, and sample width $17 \mathrm{~ms}$. 
Mesoporous $\mathrm{TiO}_{2}$ on TEC 15 conductive glass was prepared with the doctor blade method from Solaronix T paste. Similar films have been measured to be $4 \mu \mathrm{m}$ thick using the same technique. The film was sensitized from a 0.1 $\mathrm{mM}$ solution of betanin in water at $\mathrm{pH} 2.8$. The film was dried and immersed in acetonitrile with $0.2 \mathrm{M} \mathrm{NaClO}_{4}$ electrolyte. The reference electrode was silver wire. The potential of the silver wire was measured against ferricene/ferricinium after the spectrometric measurement, and the reported potentials adjusted to $\mathrm{Ag} / \mathrm{AgCl}$ reference. The spectra were measured with a Shimadzu 2501 spectrophotometer.

Transmission-mode nanosecond transient absorption spectroscopy experiments were conducted using a frequency-tripled, Q-switched Nd:YAG laser (Continuum, $20 \mathrm{~Hz}$ repetition rate, $7 \mathrm{~ns}$ pulse duration). The second harmonic $(532 \mathrm{~nm})$ was attenuated through neutral density filters and used to excite the sample. The fluence was $35 \mu \mathrm{J} / \mathrm{cm}^{2}$. The cw probe light from a Xe arc lamp was passed through various optical elements, the sample, and a monochromator to select the desired probe wavelength, before being detected by a fast photomultiplier tube and recorded by a digital oscilloscope. Averaging over at least 1,000 laser shots was used. Dynamics were recorded over 10,000 points and a $2^{\text {nd }}$ order Savitzky-Golay smoothing algorithm on 33 points was applied.

Femtosecond transient absorption spectra were recorded using femtosecond pulsed laser pump-probe spectroscopy. The pump beam (480 or $580 \mathrm{~nm}$ ) was generated with a two-stage non-collinear optical parametric amplifier (NOPA) from the $778 \mathrm{~nm}$ output of a Ti:Sa laser system with a regenerative amplifier providing $150 \mathrm{fs}$ pulses at a repetition rate of $1 \mathrm{kHz}$. The pump beam was compressed with a pair of SF-10 prisms to sub-50 fs pulses. The fluence was $20 \mu \mathrm{J} / \mathrm{cm}^{2}$. The probe consisted of a white light continuum (WLC) between 400 and $800 \mathrm{~nm}$, generated by passing a portion of the $778 \mathrm{~nm}$ amplified Ti:Sa output through a $5 \mathrm{~mm}$-thick $\mathrm{CaF}_{2}$. The probe intensity was always less than the pump intensity and the spot size was much smaller. The probe pulses were time-delayed with respect to the pump pulses using a computerized translation stage. The probe beam was split before the sample into a signal beam (transmitted through the sample and crossed with the pump beam) and a reference beam. The signal and reference were detected with a pair of spectrographs (Princeton instruments, SpectraPro 2150) equipped with 512x58 pixels back-thinned cameras (Hamamatsu S07030-0906), assembled by Entwicklungsbüro Stresing, Berlin. The pump light was chopped at half the amplifier frequency, and the transmitted signal intensity was recorded shot by shot. It was corrected for intensity fluctuations using the reference beam. The transient spectra were averaged to the desired signal-to-noise ratio (typically 3000 acquisitions). The polarization of the probe pulse was at magic angle relative to that of the pump pulse. All spectra were corrected for the chirp of the white-light probe pulse.

\section{STEADY STATE OPTICAL SPECTRA}
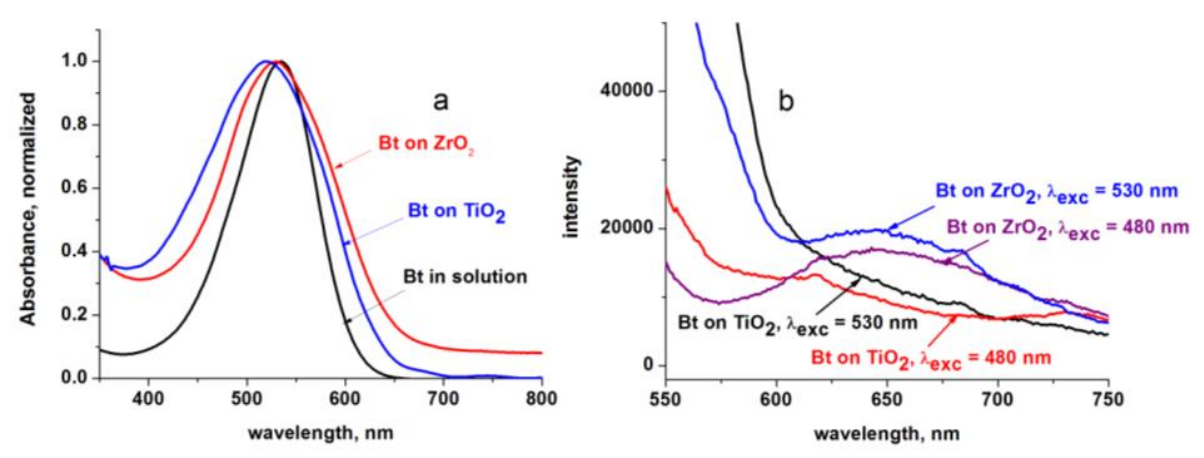

Fig. 2 a) Absorption spectrum of $\mathrm{Bt}$ in solution, on $\mathrm{TiO}_{2}$, and on $\mathrm{ZrO}_{2}$. b) Fluorescence spectrum of $\mathrm{Bt}$ on $\mathrm{TiO}_{2}$ and $\mathrm{ZrO}_{2}$ at excitations wavelengths 480 and $530 \mathrm{~nm}$.

Fig. 2a compares the normalized absorption spectrum of an aqueous solution of $\mathrm{Bt}$ to that of $2.5 \mu \mathrm{m}$ thick $\mathrm{TiO}_{2}$ and $\mathrm{ZrO}_{2}$ films sensitized from this solution. The absorption maximum of Bt shifts from $534 \mathrm{~nm}$ in solution to $529 \mathrm{~nm}$ on $\mathrm{ZrO}_{2}$ and $518 \mathrm{~nm}$ on $\mathrm{TiO}_{2}$. Fig. $2 \mathrm{~b}$ compares the weak fluorescence emission of $\mathrm{Bt}$ on $\mathrm{TiO}_{2}$ and $\mathrm{ZrO}_{2}$ at excitation wavelengths of 480 and $530 \mathrm{~nm}$. Though a scattering background from the metal oxide film is apparent in all the data, clearly 
the $\mathrm{Bt}$ emission is quenched on $\mathrm{TiO}_{2}$ but not on $\mathrm{ZrO}_{2}$, a consequence of electron injection into the conduction band of $\mathrm{TiO}_{2}$ but not $\mathrm{ZrO}_{2}$. Fluorescence data were gathered at a wavelength near the maximum absorption of $\mathrm{Bt}(530 \mathrm{~nm})$, as well as at $480 \mathrm{~nm}$ because the latter wavelength was expected to excite the strong fluorescence of any residual yellow betaxanthin pigments which are highly fluorescent. ${ }^{13}$ The coincidence of the absorption spectrum of Bt with the excitation spectrum of the emission at $610 \mathrm{~nm}$ (not shown) confirms that the weak emission is from $\mathrm{Bt}$ and not from an impurity such as residual betaxanthin.

\section{ELECTROCHEMISTRY AND SPECTROELECTROCHEMISTRY}

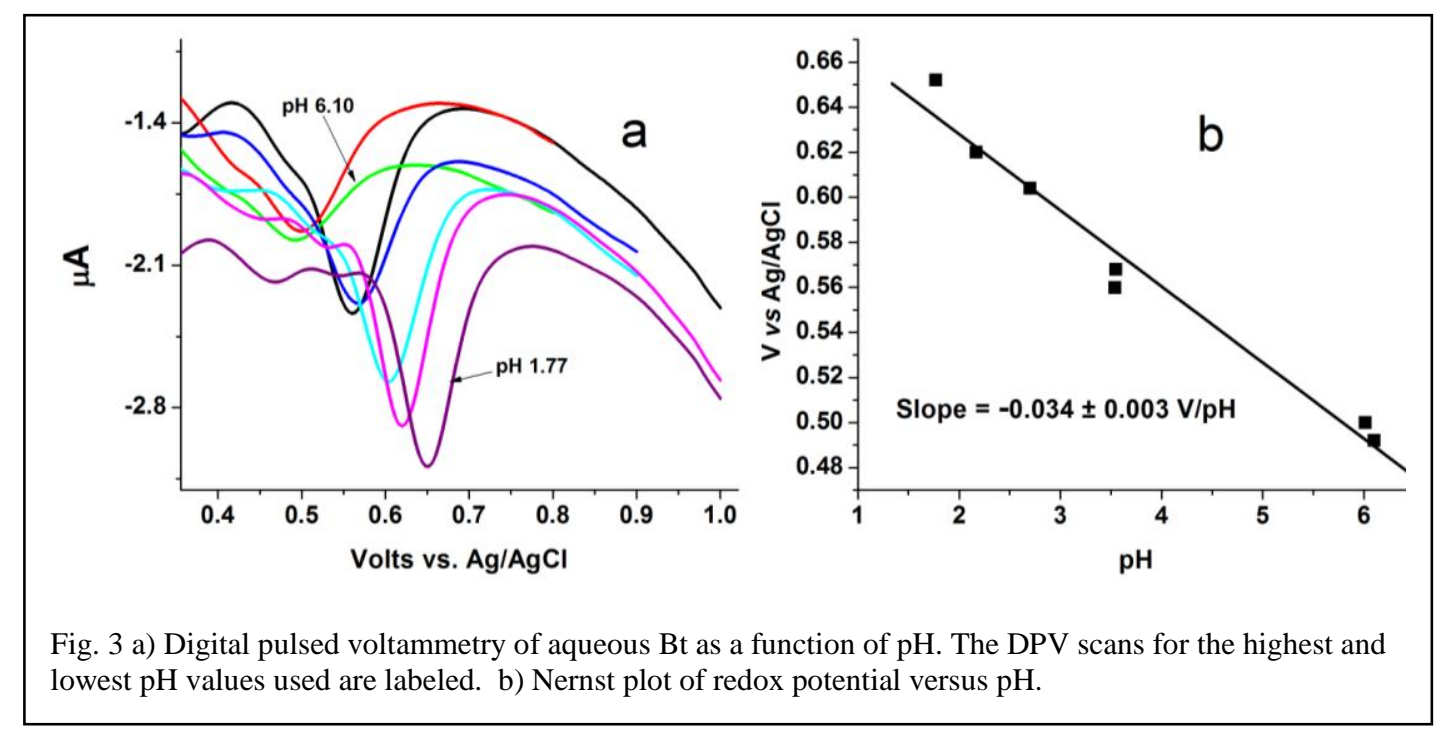

Digital pulsed voltammetry (DPV) of aqueous Bt was performed in buffered solutions as a function of $\mathrm{pH}$ and is displayed in Fig. 3. The main oxidation occurring at $0.65 \mathrm{~V}$ vs. $\mathrm{Ag} / \mathrm{AgCl}$ at the lowest $\mathrm{pH}$ (1.77) shifts to less positive potentials as the $\mathrm{pH}$ is raised, with a slope of $-0.034 \pm 0.003 \mathrm{~V} / \mathrm{pH}$. Considering that the oxidation of betanin to give $\mathrm{Bt}_{\mathrm{ox}}$ involves the loss of $m$ protons and $n$ electrons, the standard redox potential $E_{\text {red }}^{\circ}$ applies to the half-reaction $\mathrm{Bt}_{\mathrm{ox}}+n \mathrm{e}^{-}+m \mathrm{H}^{+} \rightarrow \mathrm{Bt}$. This leads to the Nernst equation:

$$
E_{\text {red }}=E_{\text {red }}^{\circ}-\frac{59 \mathrm{mV}}{n} \log \frac{[\mathrm{Bt}]}{\left[\mathrm{Bt}_{\mathrm{ox}}\right]}-\frac{m \times 59 \mathrm{mV}}{n} \mathrm{pH}
$$

Thus the observed slope of the redox potential versus $\mathrm{pH}$ suggests a one-proton, two-electron redox reaction. The scheme shown in Fig. 1, adapted from Ref. 10, suggests such a process for the oxidation of $\mathrm{Bt}$ to the quinone methide. The oxidation was found to be more facile at low $\mathrm{pH}$. Though some smaller peaks at lower potential than the main peak were observed in the DPV data at lower $\mathrm{pH}$, there was insufficient signal to track these as a function of $\mathrm{pH}$. The above results can be compared to those obtained by Butera et al. ${ }^{14}$ who found three oxidations of Bt at $0.404,0.616$. and 0.998 $\mathrm{V}$, presumably at near neutral $\mathrm{pH}$ (PBS buffer). The first of these compares to the value of $0.44 \mathrm{~V}$ obtained from the best fit to our data assuming a pH of 7. While this is in rough agreement with the first peak reported in Ref. 14, in our hands the oxidation was not observed to take place at $\mathrm{pH}$ values near 7 and above. 


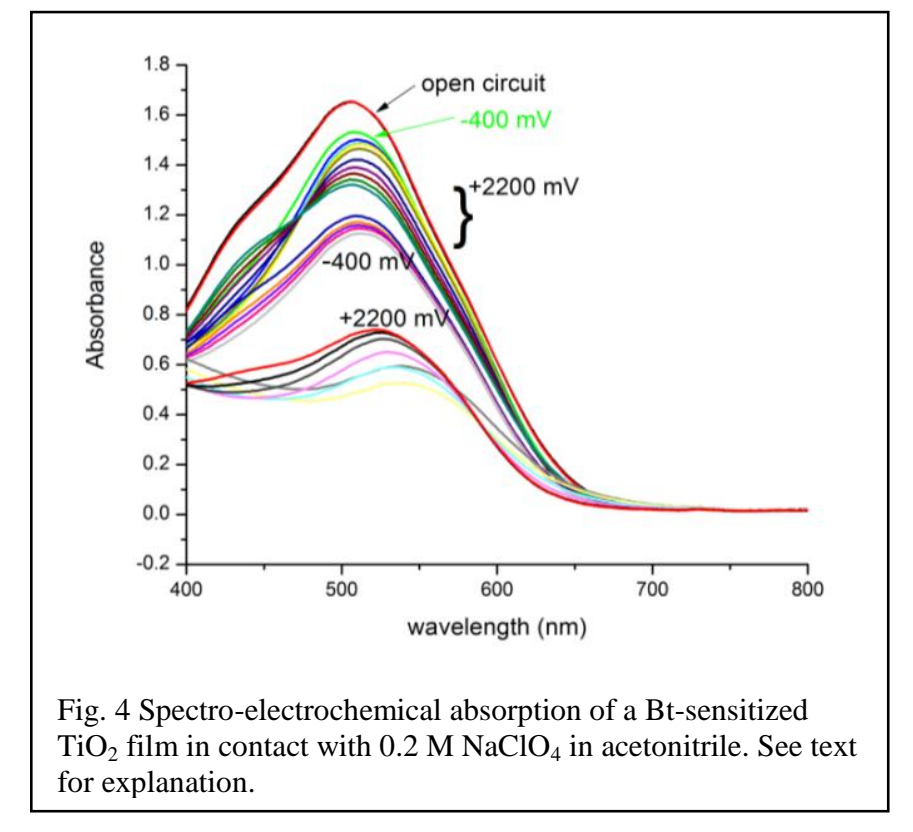

Fig. 4 shows the spectro-electrochemical absorption spectrum of $\mathrm{Bt}$ on $\mathrm{TiO}_{2}$ in contact with $0.2 \mathrm{M} \mathrm{NaClO}_{4}$ in acetonitrile as a function of applied potential versus $\mathrm{Ag} / \mathrm{AgCl}$. The data of Fig. 4 have not been normalized. Note that the initial spectrum at open circuit shows a shoulder at $\sim 450 \mathrm{~nm}$ that we believe to be pre-oxidized dye that forms under room light. Two subsequent scans at open circuit gave the same spectrum, with a maximum absorbance of 1.65 at 508 $\mathrm{nm}$. Following these two measurements, a potential of $-400 \mathrm{mV}$ was applied giving the trace shown in green in Fig. 5 . The reduction in the shoulder at $450 \mathrm{~nm}$ at this potential confirms that the oxidation is at least partly reversible. However, a decrease in the Bt absorbance peak at $508 \mathrm{~nm}$ was also observed. Following this, a potential of $+2200 \mathrm{mV}$ was applied and the spectra scanned 10 times at approximately 1 minute intervals. With each successive scan there is an increase in the blue shoulder and a decrease in the Bt peak, with an imperfect isosbestic point observed at about $475 \mathrm{~nm}$. The potential was then returned to $-400 \mathrm{mV}$ and a series of five spectra recorded. As seen in Fig. 4, this cathodic potential was capable of reducing the blue shoulder associated with the oxidation product, but the absorption at $530 \mathrm{~nm}$ does not recover fully. Finally, seven spectra were recorded while holding the film at a potential of $+2200 \mathrm{~V}$. Successive scans resulted in diminished absorbance at $530 \mathrm{~nm}$, but the spectral changes to the blue of the main peak suggest that the 450 $\mathrm{nm}$ oxidation product can undergo further oxidation.

\section{TRANSIENT ABSORPTION MEASUREMENTS}

Fig. 5 shows the global fits of the femtosecond transient absorption data of $\mathrm{Bt}$ on $\mathrm{TiO}_{2}$ and $\mathrm{ZrO}_{2}$. In each case the change in absorbance, $\Delta A$, as a function of wavelength was fit to a model of bi-exponential decay plus a long-time (residual) component. The amplitudes of the two time-dependent components plus the time-independent residual component are shown as a function of probe wavelength. In Fig. 5a is seen the result for $\mathrm{Bt}$ on $\mathrm{TiO}_{2}$ in contact with methoxypropionitrile (MPN). There are two decay components at $0.8 \mathrm{ps}$ and $6 \mathrm{ps}$. The faster decay is associated with a strong positive absorption in the blue and a negative absorption at longer wavelengths. The dip with a minimum at 530 $\mathrm{nm}$ is obviously the ground state bleach, while the negative absorbance at longer wavelengths is likely stimulated emission (SE) from Bt, in agreement with the fluorescence spectrum of the sensitized film shown in Fig. 2b. The amplitude of the slower (6 ps) decay in Fig. 5a shows a diminished positive absorption in the blue and reduced stimulated emission. The persistent bleach in the range 500 to $600 \mathrm{~nm}$ in the residual component in Fig. 5a (and in Fig. 5b) may correlate to the irreversibility observed in the spectroelectrochemical measurements discussed above. The data in Fig. $5 \mathrm{~b}$ are for $\mathrm{Bt}$ on $\mathrm{TiO}_{2}$ in the presence of reducing agent LiI. Similar decay times, $0.9 \mathrm{ps}$ and $8 \mathrm{ps}$, and similar transient features are seen for the data with and without LiI, but the relative amplitudes of the fast and slow components of the blue transient absorption (TA) are different from what was observed in the absence of LiI. Fig. 5c shows the global fit for Bt on $\mathrm{ZrO}_{2}$ in contact with MPN. Again, there is a fast $(0.8 \mathrm{ps})$ decay showing positive transients in the blue and negative $\Delta A$ at longer wavelengths, and a slower (6 ps) decay with diminished SE at red wavelengths. The flat residual component 
shown in Fig. 5c reveals that there is no apparent persistent oxidation of $\mathrm{Bt}$ on $\mathrm{ZrO}_{2}$, in accord with visual observation of the film after laser exposure and spectra recorded before and after laser exposure.
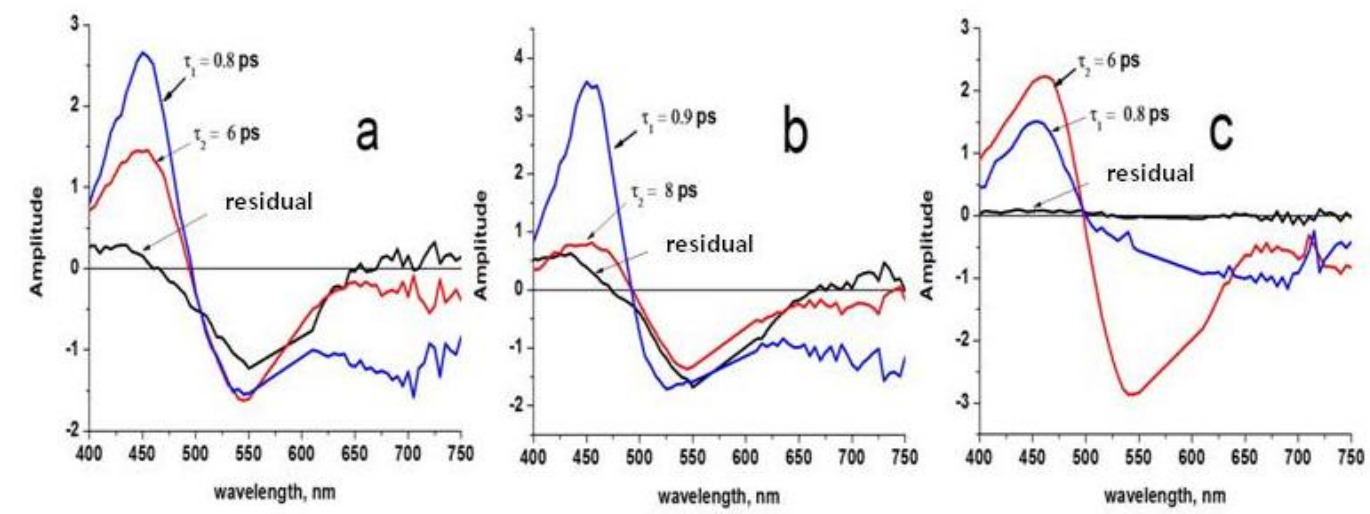

Fig. 5 Global fits of the transient absorption to a biexponential decay plus a long-time (residual) component at a pump wavelength of $580 \mathrm{~nm}$, for a) $\mathrm{Bt}$ on $\mathrm{TiO}_{2}$ in contact with MPN, b) Bt on $\mathrm{TiO}_{2}$ in contact with $0.5 \mathrm{M} \mathrm{LiI}$ in MPN, and c) Bt on $\mathrm{ZrO}_{2}$ in contact with $\mathrm{ZrO}_{2}$.

Femtosecond transient absorption spectra were also collected at a pump wavelength of $480 \mathrm{~nm}$ and are shown in Fig. 6 for a Bt-sensitized $\mathrm{TiO}_{2}$ film compared to $\mathrm{Bt}$ on $\mathrm{ZrO}_{2}$. The results are similar to what was obtained at a pump wavelength of $580 \mathrm{~nm}$. The data are fit by a bi-exponential decay with time constants of about $1 \mathrm{ps}$ and 6 to $10 \mathrm{ps}$. There is again a blue transient absorption with nearly equal amplitudes of the fast and slow decays for $\mathrm{Bt}$ on $\mathrm{ZrO}_{2}$, but a reduced amplitude of the slow TA compared to the fast for $\mathrm{Bt}_{\text {on }} \mathrm{TiO}_{2}$. Stimulated emission in the red is observed to decay mostly on the faster time scale, and the bleach in the green is observed on both time scales. One notable difference compared to the data with the $580 \mathrm{~nm}$ pump is the blue shift in the bleach in the early time amplitude. The dip at about 510 $\mathrm{nm}$ in the early time amplitude shifts to $530 \mathrm{~nm}$ in the longer time trace.
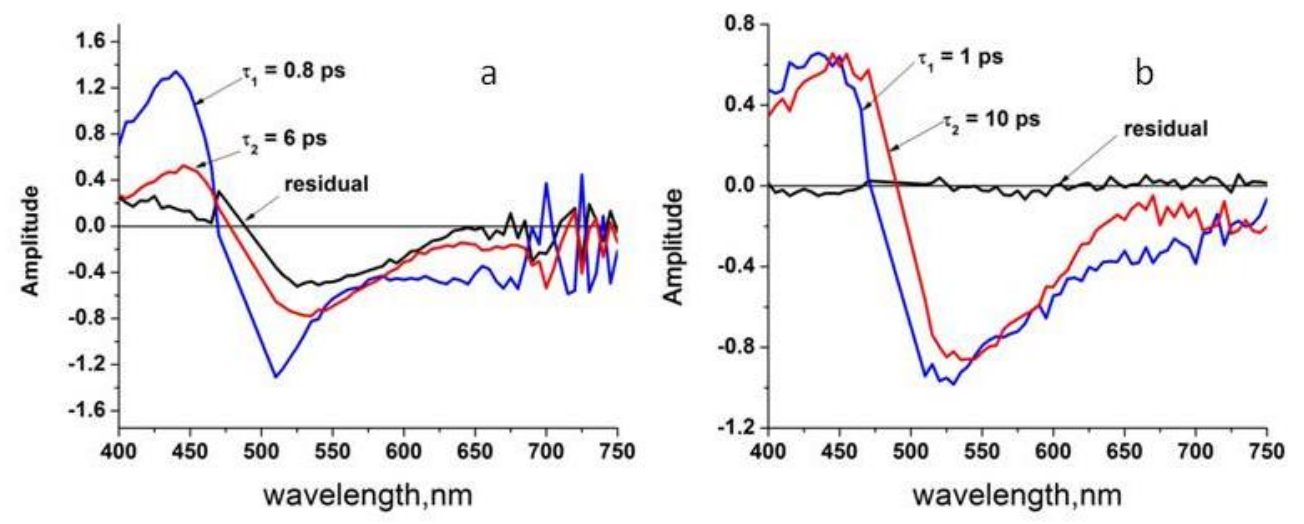

Fig. 6 Global fit of fs transient absorption to bi-exponential decay plus residual (long-lived) component at a pump wavelength of $480 \mathrm{~nm}$ for a) $\mathrm{Bt}$ on $\mathrm{TiO}_{2}$ in contact with MPN, b) Bt on $\mathrm{ZrO}_{2}$ in contact with MPN. 
Transient absorption data were also recorded on the nanosecond time scale as shown in Fig. 7. It is significant that on this time scale, no signal was observed for $\mathrm{Bt}$ on $\mathrm{ZrO}_{2}$. On $\mathrm{TiO}_{2}$, on the other hand, transient absorptions at 400$450 \mathrm{~nm}$ and $650-750 \mathrm{~nm}$ were observed and are assigned to absorption of oxidized Bt. The bleach in the green is also apparent at time scales of $200 \mathrm{~ns}$. An exponential fit to the transients at 425, 600, and $670 \mathrm{~nm}$ gave decay times of 2.0, 2.5 , and $2.2 \mu \mathrm{s}$, respectively, which are the same within the error of the measurement.

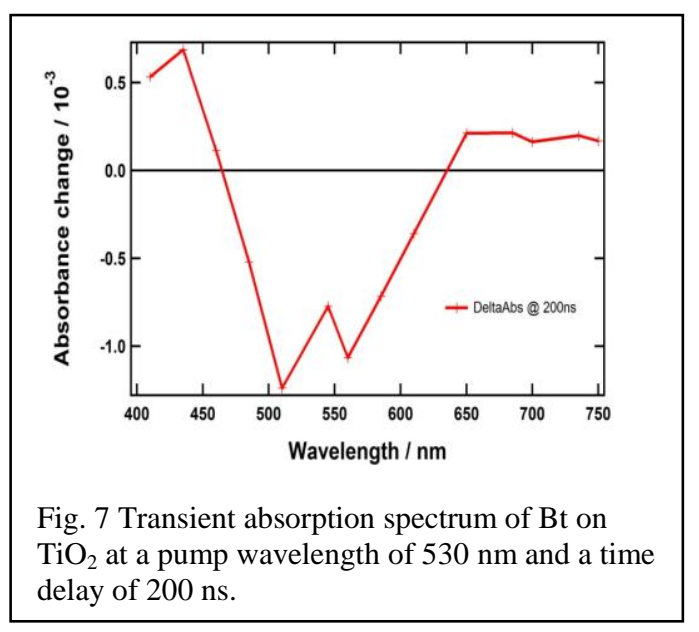

The films of $\mathrm{Bt}$ on $\mathrm{ZrO}_{2}$ were found to be quite stable during the femtosecond measurements, with no significant change in the absorption spectrum of the film. The absorption spectrum of the $\mathrm{Bt}$-sensitized $\mathrm{TiO}_{2}$ film, on the other hand, was found to undergo a significant blue shift after laser exposure. Visual inspection of the exposed parts of $\mathrm{Bt} / \mathrm{TiO}_{2}$ films revealed yellow coloration consistent with formation of oxidized dye with an absorption band in the blue.

\section{DISCUSSION}

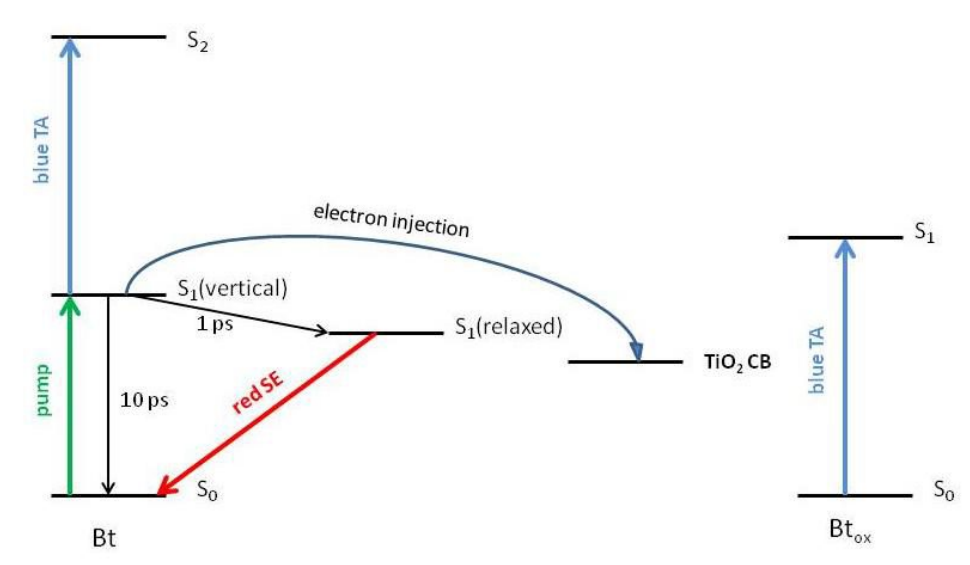

Fig. 8 Scheme describing transient events observed for $\mathrm{Bt}$ on $\mathrm{TiO}_{2}$

The femtosecond transient absorption data of Bt-sensitized $\mathrm{TiO}_{2}$ and $\mathrm{ZrO}_{2}$ consistently revealed bi-exponential decay with approximately $1 \mathrm{ps}$ and 5-8 ps time constants, referred to here as the fast and slow decay components. Common features in the femtosecond pump-probe data are the blue TA, red SE, and a green bleach, all observed on both $\mathrm{Bt} / \mathrm{TiO}_{2}$ and $\mathrm{Bt} / \mathrm{ZrO}_{2}$ films. The green bleach is also observed in the long-time component for the $\mathrm{Bt} / \mathrm{TiO}_{2}$ film but not for $\mathrm{Bt} / \mathrm{ZrO}_{2}$. The relative amplitudes of the slow and fast components of the blue TA are significantly different for the two films. On $\mathrm{ZrO}_{2}$, these amplitudes are roughly equal, while on $\mathrm{TiO}_{2}$ in the presence of MPN, the slow component of this TA has an amplitude which is only $40 \%$ that of the fast component. This ratio is further reduced to about $20 \%$ for the $\mathrm{Bt} / \mathrm{TiO}_{2}$ film in contact with LiI in MPN. Since no transient absorption is observed in the nanosecond pump-probe exper- 
iments for $\mathrm{Bt} / \mathrm{ZrO}_{2}$, the blue $(\sim 450 \mathrm{~nm})$ and red $(\sim 650-750 \mathrm{~nm})$ TA features seen for $\mathrm{Bt} / \mathrm{TiO}_{2}$ on this time scale (Fig. 7) are assigned to oxidation products. The former is certainly consistent with the oxidation product dhBt shown in Fig. 1. However, since the blue TA is also present in the fast and slow decays in the fit to the fs data for $\mathrm{Bt} / \mathrm{ZrO}{ }_{2}$, we must consider the positive absorption in the blue seen in the femtosecond experiments can also result from excited state absorption, i.e. the transition $\mathrm{S}_{1} \rightarrow \mathrm{S}_{2}$. The red SE, observed for $\mathrm{Bt}$ on both $\mathrm{TiO}_{2}$ and $\mathrm{ZrO}_{2}$, is coincident with the fluorescence spectrum of Bt.

A possible scheme explaining these results is presented in Fig. 8. Consider first the transient events taking place on $\mathrm{ZrO}_{2}$, where we assume there is no photo-oxidation of $\mathrm{Bt}$ on any time scale. The bimodal decay of the blue TA could be a consequence of fast thermal relaxation of the vertical (Franck-Condon) excited state to the relaxed excited electronic state, accompanied by slower parallel relaxation of the vertical excited state directly to the ground state. This would explain the weak negative absorption (from SE) in the red at longer time scales, and the observation that the negative absorption in the green decays on both time scales as the ground state population recovers. Given that the fluorescence quantum yield of $\mathrm{Bt}$ in aqueous solution is on the order of $\varphi_{f} \approx 0.001$ (unpublished work), and with $\tau_{\text {rad }}$ on the order of $5 \mathrm{~ns}$ (typical value for a strongly allowed $S_{0} \rightarrow S_{1}$ transition with oscillator strength near unity), a nonradiative relaxation time $\tau_{\text {rad }}$ of about 5 ps is expected in accord with our observations.

Interpretation of the femtosecond pump-probe data for $\mathrm{Bt}$ on $\mathrm{TiO}_{2}$ is complicated by the apparent coincidence of the absorption of oxidized $\mathrm{Bt}\left(\mathrm{Bt}_{\mathrm{ox}}\right)$ with the $\mathrm{S}_{1} \rightarrow \mathrm{S}_{2} \mathrm{TA}$, which both occur at about $450 \mathrm{~nm}$. The fast and slow time constants of the bi-exponential relaxation are not significantly different for $\mathrm{Bt}$ on $\mathrm{TiO}_{2}$ versus $\mathrm{ZrO}_{2}$, however, the relative amplitudes vary greatly. For $\mathrm{Bt} / \mathrm{TiO}_{2}$ in the presence of MPN, the amplitude of the slow component of the blue TA is about $40 \%$ of that of the fast component. Excited state electron injection into the conduction band of $\mathrm{TiO}_{2}$ can be reasonably assumed to take place from the vertical excited state, leading to greater amplitude of the fast decay of the excited electronic state. However, if the result is offset by the appearance of positive blue $\mathrm{TA}$ from $\mathrm{Bt}_{\mathrm{ox}}$, we would see increased amplitude of the fast decaying blue TA only if the oscillator strength of $\mathrm{Bt}_{\mathrm{ox}}$ is less than that of the $\mathrm{S}_{1} \rightarrow \mathrm{S}_{2}$ transition. Only the relative amplitudes are meaningful, and the additional fast relaxation channel resulting from electron injection from the vertical state reduces the relative amount of relaxed excited state dye and thus the relative amplitude of the slowly relaxing blue TA.

In the presence of LiI (Fig. 5b), we expected to see changes in the dynamics that reflect regeneration of ground state $\mathrm{Bt}$ from $\mathrm{Bt}_{\mathrm{ox}}$, e.g., faster ground state recovery. This was not apparent, but we do observe that the amplitude of the slow decay of the blue TA is reduced to about $20 \%$ of that of the fast decay. This effect could result from the well-known effect of $\mathrm{Li}^{+}$on the $\mathrm{TiO}_{2}$ conduction band. A lower energy conduction band edge in the presence of $\mathrm{Li}^{+}$could account for increased amplitude of the fast decay resulting from electron injection, the driving force for which is greater than in the absence of $\mathrm{Li}^{+}$. Thus, compared to $\mathrm{Bt}$ on $\mathrm{ZrO}_{2}$ and on $\mathrm{TiO}_{2}$ in the absence of LiI, the relative amplitude of the slowly relaxing blue TA is further reduced as electron injection from the vertical excited state is enhanced.

The nanosecond transient absorption data reveal a red absorption band from an oxidized form of Bt. This does not appear to be an additional transition of the same species that gives rise to the $450 \mathrm{~nm}$ absorption band, since it is not seen in the steady-state spectra of oxidized dye. A positive red TA was not seen in the femtosecond data, but might have been offset by the negative $\Delta A$ resulting from SE. DFT calculations are presently underway to investigate the possibility that the red TA observed in Fig. 7 is an intermediate such as the quinone methide shown in Fig. 1, a degradation product, or a one-electron semiquinone oxidation product. The results of these calculations will be reported in a separate publication.

\section{CONCLUSIONS}

Transient positive absorption at $450 \mathrm{~nm}$ in femtosecond pump-probe measurements of $\mathrm{Bt}$ on $\mathrm{TiO}_{2}$ results from excited state absorption which coincides with the two-electron oxidation product 2-decarboxy-2,3-dehydrobetanin. Relative amplitudes of the fast and slow decay components of the blue TA are consistent with increased efficiency of electron injection in the presence of LiI. Nanosecond data of $\mathrm{Bt}_{\text {on }} \mathrm{TiO}_{2}$ is entirely due to oxidation products, and reveals the two-electron oxidation product and an as-yet unknown intermediate that absorbs in the red. The results of analysis of the nanosecond data will be presented in a future publication along with DFT calculations of potential intermediates in the oxidation of Bt. 


\section{ACKNOWLEDGMENTS}

The support of the National Science Foundation through grant DMR 1305592 is gratefully acknowledged. FJK and JLM thank Jacques Moser and his group members for their gracious hospitality during their stay at EPFL.

\section{REFERENCES}

[1] O’Regan, B. and Grätzel, M. "A low-cost, high-efficiency solar cell based on dye-sensitized colloidal titanium dioxide films,” Nature 35 (6356), 737-40 (1991).

[2] Hagfeldt, A., Boschloo, G., Sun, L., Kloo, L. and Pettersson, H., "Dye-sensitized solar cells," Chem. Rev. 110(11), 6595-6663 (2010).

[3] Zhang, D., Lanier, S., Downing, J. A., Avent, J., Lum, J. and McHale, J. L. "Betalain pigments for dye-sensitized solar cells," J. Photochem. Photobio. A 195(1), 72-80 (2008).

[4] Sandquist, C., McHale, J. "Improved efficiency of betanin-based dye-sensitized solar cells," J. Photochem. Photobio. A 221(1), 90-97 (2011).

[5] Hernandez-Martinez, A. R., Estevez, M., Varga, S., Quintanilla, F. and Rodriguez, R. "New dye-sensitized solar cells obtained from extracted brachts of bougainvillea glabra and spectabilis betalain pigments by different purification process,” Int. J. Mol. Science 12, 5565-5576 (2011).

[6] Calogero, G., Yum, J.-H., Sinopoli, A., Di Marco, G., Grätzel, M. and Nazeeruddin, M. K. “Anthocyanins and betalains as light-harvesting pigments for dye-sensitized solar cells," Solar Energy 86(5), 1562-1575 (2012).

[7] Calogero, G., di Marco, G., Caramori, S., Cazzanti, S., Argazzi, R. and Bignozzi, C. A. "Natural dye-sensitizers for photoelectrochemical cells," Energy Environ. Science 2(11), 1162-1172 (2009).

[8] Smestad, G. "Testing of dye-sensitized $\mathrm{TiO}_{2}$ cells II: Theoretical voltage output and photoluminescence efficiencies," Solar Energy Mater. Solar Cells 32(3), 272-288 (1994).

[9] Gandía-Herrero, F., Escribano, J. and García-Carmona, F. "The role of phenolic hydroxyl groups in the free radical scavenging ability of betalains," J. Nat. Prod. 72(6), 1142-1146 (2009).

[10] Wybraniec, S., Stalica, P., Spórna, A., Nemzer, B., Pietrzkowski, Z. and Michałowski, T. "Anitoxidant activity of betanidin: Electrochemical study in aqueous media,” J. Agric. Food Chem. 59(22), 12163-12170 (2011).

[11] Wybraniec, S. and Michałowski, T. "New pathways of betanidin and betanin enzymatic oxidation," J. Agric. Food Chem. 59(17), 9612-9622 (2011).

[12] Wybraniec, S., Starzak, K., Skopińska, A., Nemzer, B., Pietrzkowski, Z. and Michałowski, T. "Studies on nonenzymatic oxidation mechanisms in neobetanin, betanin, and decarboxylated betanins," J. Agric. Food Chem. 61(26), 6465-6476 (2013).

[13] Gandía-Herrero, F.; García-Carmona, F.; Escribano, J. "Fluorescent pigments: new perspectives in betalain research and applications," Food Research Int. 38(8-9), 879-884 (2005).

[14] Butera, D., Tesoriere, L., Di Gaudio, F., Bongiorno, A., Allegra, M., Pintaudi, A.M., Kohen, R. and Livrea, M.A. "Antioxidant activities of sicilian prickly pear (Opuntia ficus indica) fruit extracts and reducing properties of its betalains: betanin and indicaxanthin” J. Agric. Food Chem. 50(23), 6895-6901 (2002). 